\title{
How asteroseismology can constrain the global parameters of solar-like star models
}

\author{
N. Ozel • M.-A. Dupret • A. Baglin
}

Received: 9 October 2009 / Accepted: 25 January 2010 / Published online: 13 April 2010

(C) Springer Science+Business Media B.V. 2010

\begin{abstract}
In the previous years, p-mode oscillations (pressure oscillations stochastically excited by convection) have been detected in several solar-like stars thanks to the groundbased spectroscopic and space spectroscopic and photometric observations. We study the importance of seismic constraints on stellar modeling and the impact of their accuracy on reducing the uncertainties of global stellar parameters (i.e. mass, age, etc.). We use the Singular Value Decomposition (SVD) method to analyze the sensitivity of stellar models to seismic constraints. In this context, we construct a grid of evolutionary sequences for solar-like stars with varying age and mass. Around each model of this grid, we evaluate the partial derivatives with respect to a large set of free parameters: mass $\mathcal{M}$, age $\tau$, mixing-length parameter $\alpha$, initial helium abundance $Y_{0}$, and initial metallicity $Z / X_{0}$. Masses between 0.9 and $1.55 M_{\odot}$ and central hydrogen abundances from $\mathrm{Xc}=0.7$ to 0.05 have been considered in this study.
\end{abstract}

Keywords Solar-like stars: fundamental parameters

\section{Introduction}

Observations of solar p-mode oscillations have greatly improved solar modeling and resulted in great progress in understanding the internal structure of the Sun. Successes in the solar case (e.g., Christensen-Dalsgaard 2002) have

N. Ozel $(\bowtie) \cdot$ A. Baglin

Observatoire de Paris, LESIA, CNRS UMR 8109, 92195

Meudon, France

e-mail: Nesibe.Ozel@obspm.fr

M.-A. Dupret

Institut d'Astrophysique et de Géophysique de l'Université de Liège, Allée du 6 Août 17, 4000 Liège, Belgium suggested that analogous progress might be made if one could obtained frequency and amplitude information about such oscillations on other solar-like stars. The detection of p-mode oscillations in solar-like stars from ground-based observations carried out for example by CORALIE and HARPS spectrographs and space observations by CoRoT and Kepler satellites have opened the possibility of taking this success further.

The precise measurement of individual oscillation frequencies allows us to obtain detailed information on the structure of stellar interior, and this thus provides the constraints on the basic physical processes (convection, rotation, mixing) to test the stellar structure theory and to analyze the inferred stellar parameters at a high level of precision.

\section{Methodology}

The general mathematical approach of this study employs a $\chi^{2}$ minimization technique based on the Singular Value Decomposition (SVD) method. Here we provide a general description of the method to enable the reader to understand the work. A more detailed explanation of the technique can be found in Brown et al. (1994), Creevey et al. (2007) and Ozel et al. (2010).

Let $y_{i}, i=1, \ldots, n$, be the model functions, and $x_{j}, j=$ $1, \ldots, m$, with $n>m$ be the parameters that define the model function. The model functions which we call observables are the theoretical predictions for observed quantities $y_{\text {obs }, i}$ such as effective temperature $T_{\text {eff }}$, luminosity $L / L_{\odot}$, etc. The model function must allow for computation of all the observables $y_{i}$, given values for the parameters $x_{j}$. Given a set of $n$ measurements $y_{\mathrm{obs}, i}$ with associated error $\left(\sigma_{i}\right)$, we determine the reference model, depending non-linearly on 
a set of $m$ parameters $x_{j}$ (e.g. mass, age, etc.) by minimizing the differences between observational constraints $y_{\mathrm{obs}, i}$ and model predictions (or observables) $y_{i}$ in the least square sense:

$\chi^{2}=\left\|\frac{\mathbf{y}_{\text {obs }}-\mathbf{y}(\mathbf{x})}{\sigma}\right\|^{2}$.

Linearizing the model function around a reference set of parameters, $x_{j 0}$, we can write

$y_{i}=y_{i 0}+\sum_{j=1}^{M} \frac{\partial y_{i}}{\partial x_{j}} \delta x_{j}$,

where $\delta x_{j}=x_{j}-x_{j 0}, y_{i 0}$ is the set of observables resulting from the reference set of parameters $x_{j 0}$, and the derivatives $\partial y_{i} / \partial x_{j}$ are evaluated at $x_{j}=x_{j 0}$. After substituting the expression (2) into (1), the $\chi^{2}$ minimization problem consists of choosing $\delta \mathbf{x}$ so that

$\chi^{2}=\|\mathbf{D} \delta \mathbf{x}-\delta \mathbf{B}\|$

is a minimum. $\delta B_{i}=\left(y_{i}-y_{i 0}\right) / \sigma_{i}$ and $\mathbf{D}$ is called design matrix or derivative matrix following the work of Brown et al. (1994):

$D_{i j}=\frac{1}{\sigma_{i}} \frac{\partial y_{i}}{\partial x_{j}}$.

This matrix relates small changes in the parameters to corresponding changes in the observables. There are several different local approach available for finding this minimum, such as the Levenberg-Marquardt algorithm or the Singular Value Decomposition Method (SVD).

Supposing that $\mathbf{x}_{0}$ is the true set of parameters of model functions that minimize the $\chi^{2}$ fitting function, the behavior of $\chi^{2}$ around its minimum can be expressed thus:

$\Delta \chi^{2}=\chi^{2}-\chi_{\min }^{2}=\|\mathbf{D} \delta \mathbf{x}\|$,

where $\chi_{\min }^{2}$ is a minimum at $\mathbf{x}_{\mathbf{0}}$. Using the SVD method $\left(D_{n \times m}=U_{n \times m} W_{m \times m} V_{m \times m}^{T}\right)$ and after some manipulation, one finds an $m$ dimensional ellipsoidal equation:

$\Delta \chi^{2}=\frac{\left(\mathbf{V}^{(\mathbf{1})} \cdot \delta \mathbf{x}\right)^{2}}{W_{1}^{-2}}+\frac{\left(\mathbf{V}^{(\mathbf{2})} \cdot \delta \mathbf{x}\right)^{2}}{W_{2}^{-2}}+\cdots+\frac{\left(\mathbf{V}^{(\mathbf{m})} \cdot \delta \mathbf{x}\right)^{2}}{W_{m}^{-2}}$.

The linear combinations of each column of the $\mathbf{V}$ matrix are the principal axes of the error ellipsoid $\Delta \chi^{2}=1$. The length of these axes is determined by corresponding singular values $W^{-1}$. The major advantage of the method adopted here is that it is more convenient to analyze the error ellipsoid in the parameter space and gives us the information concerning the origin of uncertainties on the obtained parameters.
The estimation of the covariances matrix of the free parameters due to the measurement errors on the $n$ observational constraints is expressed by

$\operatorname{Cov}\left(\delta x_{j}, \delta x_{k}\right)=\sum_{i}^{N} \frac{V_{j i} V_{k i}}{W_{i}^{2}}$.

The diagonal elements of Cov give the expected uncertainty in each of the parameters:

$\epsilon\left(x_{j}\right)=\sqrt{\operatorname{Cov}\left(\delta x_{j}^{2}\right)}=\sum_{i}^{N}\left(\frac{V_{j i}}{W_{i}}\right)^{2}$.

\section{Grid of models}

We describe a solar-like star with five adjustable parameters: mass $\mathcal{M}$, initial chemical composition reduced to two parameters (initial helium $Y_{0}$, and metallicity $Z / X_{0}$ ), stage of evolution or age $\tau$, and mixing-length parameter $\alpha$ which describes stellar convection in the mixing-length formulation.

We use the CESAM2k stellar evolution code (Morel and Lebreton 2008) for stellar structure and evolution and adiabatic pulsation code LOSC (Scuflaire et al. 2008) to calculate nonradial oscillation frequencies. For each model evolved with the parameters $\tau, \mathcal{M}, \alpha, Y_{0}$, and $Z / X_{0}$, the CESAM2k code produces physical parameters, which are then used to calculate the oscillation frequencies with LOSC. The outputs from the models computed in this work are the classical observables like effective temperature $T_{\text {eff }}$, luminosity $L / L_{\odot}$, and seismic quantities such as the mean and individual large and small frequency separations $\bar{\Delta} \nu_{0}$, $\bar{\delta} v_{02}, \Delta v_{0, i}$, and $\delta v_{02, i}$, respectively, for $n=15-25$ and $l=0-3$, which correspond to detectable modes.

The computation of stellar models was preformed assuming OPAL equation of state and OPAL96 opacities (Iglesias and Rogers 1996), completed at low temperatures with the opacities of Alexander and Ferguson (1994). The adopted physical description for the convective zone is the standard Mixing-Length Theory (MLT, Böhm-Vitense 1958). Diffusion is not included in the computation of our models. This study concentrates on stellar models of solar-like stars. The reference models are computed with the parameters listed in Table 1 . The observables and their expected standard errors are given in Table 2.

To construct the derivative matrix $D$ of the model, we vary each of the parameters $\left(x_{j}=\tau, \alpha, \mathcal{M}, Y_{0}, Z / X_{0}\right)$. Each derivative has been computed from differences of $\delta x$ centered on the reference parameter values $x_{j 0}$ given in Table 1 , i.e. $x_{j 0} \pm \delta x$. The interval $\delta x$ has to be sufficiently small such that the linear approximation is still valid, but also large 
Table 1 Parameters of the reference models

\begin{tabular}{ll}
\hline Parameter & Value \\
\hline$\tau$ & $13-1.8(\mathrm{Gyr})$ \\
$\mathcal{M}$ & $0.9-1.55 M_{\odot}$ \\
$\alpha$ & 1.61 \\
$Y_{0}$ & 0.2773 \\
$Z / X_{0}$ & 0.0245 \\
\hline
\end{tabular}

Table 2 The observables $y_{i, \text { obs }}$ and their expected errors $\delta y / y_{i, \text { obs }}$ are expressed as the relative errors in percent

\begin{tabular}{ll}
\hline$y_{\mathrm{obs}, i}$ & $\delta \ln y_{\mathrm{obs}, i}(\%)$ \\
\hline$T_{\text {eff }}$ & 0.86 \\
$L / L_{\odot}$ & 1.97 \\
$\bar{\Delta} \nu_{0}$ & 0.1 \\
$\bar{\delta} v_{02}$ & 12.5 \\
\hline
\end{tabular}

Table 3 The derivative intervals $\left(\delta x_{j}\right)$ and time step $(t s)$ between two model calculation used for reference models (RM)

\begin{tabular}{lllllll}
\hline $\mathrm{RM}\left(M_{\odot}\right)$ & $t s(\mathrm{Myr})$ & $\delta \tau$ & $\delta \alpha$ & $\delta \mathcal{M}$ & $\delta Y_{0}$ & $\delta Z / X_{0}$ \\
\hline 0.9 & 50 & 20 & 0.05 & 0.01 & 0.005 & 0.001 \\
0.95 & 40 & 20 & 0.04 & 0.01 & 0.005 & 0.001 \\
1 & 40 & 20 & 0.04 & 0.01 & 0.004 & 0.001 \\
1.05 & 40 & 20 & 0.05 & 0.01 & 0.004 & 0.001 \\
1.1 & 40 & 20 & 0.06 & 0.01 & 0.003 & 0.001 \\
1.25 & 20 & 20 & 0.1 & 0.01 & 0.004 & 0.001 \\
1.31 & 20 & 20 & 0.1 & 0.01 & 0.004 & 0.001 \\
1.4 & 20 & 20 & 0.3 & 0.01 & 0.004 & 0.001 \\
1.55 & 20 & 20 & 0.5 & 0.01 & 0.004 & 0.001 \\
\hline
\end{tabular}

enough to guarantee sufficient numerical accuracy. The increments $\delta x_{j}$ and time step used for the grid of reference stellar models are reported in Table 3.

\section{Results}

To investigate the role of asteroseismology in constraining the global stellar parameters, we take stellar mass and age $\tau$ as free parameters, assuming that the other parameters are precisely known.

To analyze the potential diagnostic of the oscillation information in determining the global stellar parameters of solar-like stars, we considered three sets of observables: $Y_{1}=L / L_{\odot}, T_{\text {eff }} ; Y_{2}=L / L_{\odot}, T_{\text {eff }}, \bar{\Delta} v_{0}, \bar{\delta} v_{02} ; Y_{3}=$ $L / L_{\odot}, T_{\text {eff }}, \Delta v_{0, i}, \delta v_{02, i}$.

In Fig. 1, the uncertainties on the $\mathcal{M}$ and $\tau$ are shown as a function of the mass of the stellar models for the central hydrogen abundance $\mathrm{Xc}=0.6,0.4$, and 0.2. In Fig. 1, the red cases in each panel does not include any seismic information, but only classical observables $\left(Y_{1}\right)$. The green cases include the mean large $\bar{\Delta} \nu_{0}$ and small $\bar{\delta} \nu_{02}$ separations as well as the classical observables $\left(Y_{2}\right)$. The blue cases include the individual large $\Delta \nu_{0, i}$ and small $\delta v_{02, i}$ separations instead of their mean values $\left(Y_{3}\right)$.

According to the asymptotic theory (Vandakurov 1967; Tassoul 1980), the large separation $\Delta v_{0}$ is equal to the reciprocal travel time across the star and it scales as the mean density $\bar{\rho} \propto M / R^{3}$. For stars on the main sequence (MS), the mean density decreases as mass increases and at a given mass it decreases as evolution proceeds. Thus, the large separation is quite sensitive to the mass and to the evolutionary state on the MS. On the other hand, the small separation $\delta_{02}$ is determined predominantly by the sound speed gradient weighted by $1 / r$ in the core. The sensitivity of the small separation to the changes in the core structure makes this quantity a measure of the evolutionary state of the star.

For the solar-like stars without an identified oscillation mode, the red cases $\left(Y_{1}\right)$ in Fig. 1, the age and mass are poorly constrained on the MS of low mass stars. Including the mean large and small separations $\bar{\Delta} \nu_{0}, \bar{\delta} \nu_{02}$ (the green cases, $Y_{2}$ ), the precision on all stellar parameters increases significantly. Using the individual separations $\Delta v_{0, i}, \delta v_{02, i}$ (the blue cases, $Y_{3}$ ) instead of the mean large and small separations $\left(\bar{\Delta} v_{0}, \bar{\delta} v_{02}\right)$, we obtain a better precision on the mass and the age of solar-like stars. The precision of the parameters increases as the mass increases.

We have assumed that the relative errors on the observables in the grid are constant. However, this is not necessarily realistic. For example, the errors on effective temperature $T_{\text {eff }}$ and luminosity $L / L_{\odot}$ are generally larger for high mass stars. We have not taken this into account in the analysis. Also, the effect of convection on the spectra is more uncertain for more massive stars. So, it would be wrong to conclude that in general more massive stars can be constrained better than the less massive ones.

We remark that there is little difference between the green cases (the mean separations) and blue ones (the individual separations). This indicates that the individual separations do not bring much more information than the mean separations, because we are not very far from the asymptotic regime for small masses. However, it is not the case for higher masses.

With the accuracy of a few $0.1 \mu \mathrm{Hz}$ on the observed frequencies (the accuracy achievable by CoRoT and Kepler satellites), the mass and age of a solar-like star could be determined with an accuracy of about $2 \%$ and $10 \%$, respectively. 
Fig. 1 The uncertainties on the mass $\epsilon(\mathcal{M})$ (left panels) and age parameter $\epsilon(\tau)$ (right panels) parameters as a function of stellar mass. The values of the central hydrogen abundance are indicated in each panel. The red case does not include any seismic information. The green cases include the mean large $\bar{\Delta} v_{0}$ and small $\bar{\delta} v_{02}$ separation information. The blue cases include the individual large $\Delta v_{0, i}$ and small $\delta v_{02, i}$ separations instead of their mean values

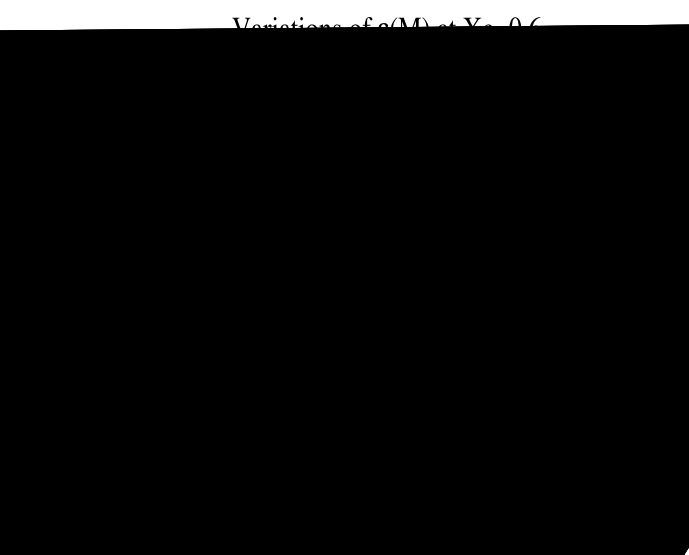

Variations of $\varepsilon(\tau)$ at

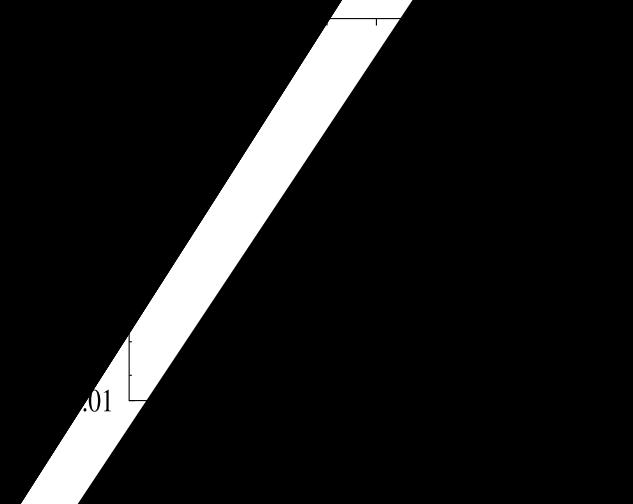


els. The computation of stellar model depends on assumptions about the physical properties of stellar plasma such as the equation of state, the opacity, the rate of nuclear reactions, etc. A more systematic analysis should also explore the effects of changes in the physical assumptions, i.e. different convection models, core overshooting and rotation, and so on. These uncertainties in current stellar evolution theory will increase the degeneracy of stellar parameters.

In this work, we considered a small number of free stellar parameters $(\mathcal{M} \tau)$, and the conclusion could be different if it increased. Thus, to generalize this conclusion, we will consider more parameters such as the mixing-length parameter for convection $\alpha$, the initial chemical composition (helium abundance $Y_{0}$, metallicity $\left.Z / X_{0}\right)$.

In conclusion, our assessment of the accuracy achievable in the determination of the stellar parameters shows that a higher accuracy in seismic information leads to a better precision in the stellar parameters. This will be the case for all stars observed for CoRoT and Kepler and, therefore, it is very important to assess the importance of the seismic data.

\section{References}

Alexander, D.R., Ferguson, J.W.: Low-temperature Rosseland opacities. Astrophys. J. 437, 879-891 (1994)

Böhm-Vitense, E.: Über die Wasserstoffkonvektionszone in Sternen verschiedener Effektivtemperaturen und Leuchtkräfte. Mit 5 Textabbildungen. Z. Astrophys. 46, 108 (1958)

Brown, T.M., Christensen-Dalsgaard, J., Weibel-Mihalas, B., Gilliland, R.L.: The effectiveness of oscillation frequencies in constraining stellar model parameters. Astrophys. J. 427, 1013-1034 (1994)

Christensen-Dalsgaard, J.: Solar g-mode oscillations. Int. J. Mod. Phys. D 11, 995-1009 (2002)

Creevey, O.L., Monteiro, M.J.P.F.G., Metcalfe, T.S., Brown, T.M., Jiménez-Reyes, S.J., Belmonte, J.A.: The complementary roles of interferometry and asteroseismology in determining the mass of solar-type stars. Astrophys. J. 659, 616-625 (2007)

Iglesias, C.A., Rogers, F.J.: Updated opal opacities. Astrophys. J. 464, 943 (1996)

Morel, P., Lebreton, Y.: CESAM: a free code for stellar evolution calculations. Astrophys. Space Sci. 316, 61-73 (2008)

Ozel, N., Dupret, M.A., Michel, E., Baglin, A.: Astron. Astrophys. (2010, submitted)

Scuflaire, R., Montalbán, J., Théado, S., Bourge, P.-O., Miglio, A., Godart, M., Thoul, A., Noels, A.: The Liège oscillation code. Astrophys. Space Sci. 316, 149-154 (2008)

Tassoul, M.: Asymptotic approximations for stellar nonradial pulsations. Astrophys. J. Suppl. Ser. 43, 469-490 (1980)

Vandakurov, Y.V.: The frequency distribution of stellar oscillations. Astron. Zh. 44, 786 (1967) 